\title{
Recent developments related to multifunctional ferroelectric for room-temperature applications
}

\author{
ZHANG Yan ${ }^{1,2^{*}}$ \\ ${ }^{1}$ School of Physical Electronics, University of Electronic Science and Technology of China, Chengdu 610054, China; \\ ${ }^{2}$ Institute of Theoretical Physics, Lanzhou University, Lanzhou 730000, China
}

Received November 28, 2015; accepted December 24, 2015

Ferroelectric materials are good candidates for coupling properties of other functions, such as mechanical, superconductivity, and magnetism. Recent publications have reported important approaches of bulk and nanostructure ferroelectric materials at room temperature [1-5]. For bulk and nanostructure ferroelectric materials, applications at room temperature are technical challenges in information storage and nanoelectronics. The reported approaches provide a few new directions for research on low-power [1] and high-density storage [2] of nonvolatile ferroelectric memories, multifunctional nanoelectronics [2-4], discovery of new physical phenomena [1-3], and energy conversion applications [5].

Magnetoelectric multiferroic materials enable fast electrical writing and magnetic reading, and can thus be applied to non-volatile random access memory. However, fast electrical writing and magnetic reading exist more difficulties in single crystalline structure bulk materials because ferroelectricity need closed-shell $d^{0}$ or $s^{2}$ cation and ferromagnetic need open-shell $d^{n}$ with unpaired electron. Although previous works have reported composite multiferroics at room temperature by multiphase mixtures of magnetic and ferroelectric materials [6], the magnetoelectric coupling effect will prevent complete switch of ferroelectricity. In a recent issue of Nature, researchers at the University of Liverpool, Trinity College Dublin, and West Virginia University reported tunable multiferroic materials at room temperature in a bulk perovskite oxide [1]. The authors constructed a percolating network of magnetic ions with strong superexchange interactions, and this network has a structural scaffold with polar lattice symmetries at a morphotropic phase

*Corresponding author (email: zhangyan@uestc.edu.cn) boundary. This strategy overcomes synthesizing difficulties for tunable multiferroic materials and provides a new direction for applications in low-power and high-density information storage.

Ferroelectric can be coupled to other functional properties. For example, the conduction, dielectric, and magnetic properties of ferroelectrics can be changed by strain. Theoretical works have predicted that alkaline-earth manganites that have the perovskite structure with larger-than-equilibrium lattice parameters exhibit ferroelectricity. For bulk $\mathrm{SrMnO}_{3}$, ferroelectricity is verified by partially replacing $\mathrm{Sr}$ with Ba. Density functional theory (DFT) calculations predicted that epitaxial $\mathrm{SrMnO}_{3}$ films will exhibit polarization under $>1 \%$ epitaxial tensile strain. Researchers in Switzerland and Spain reported that strain increases the concentration of oxygen vacancies, which couple to the polar domain walls in $\mathrm{SrMnO}_{3}$ thin films (thickness of $20 \mathrm{~nm}$ ) [2]. By DFT calculations and experimental measurements, they found that the local structures conducting polar nanodomains at room-temperature are embedded in insulating domain boundaries, which can form "nanocapacitor" domains. The stable capacitance offers "nanobits," which have potential applications in high-density information storage at room temperature.

Ferroelectric materials have important nanoelectronic applications at room temperature [3]. Many researchers have demonstrated that nanostructures will enhance the performance of nanodevices [2-4]. However, it is a longstanding notion that ferroelectricity will fade in a few nanometers scale [3]. Previous studies developed several strategies to overcome the low dimension difficulties of ferroelectricity have been proposed, e.g., by adopting strain and interface engineering. At the University of Wiscon- 
sin-Madison and University of Nebraska, researchers recently developed a new mechanism to generate roomtemperature ferroelectricity while reduce the film thickness. The results of their theoretical calculations and experiments presented evidence of ferroelectricity in the strain-free epitaxial nanofilm of strontium titanate $\left(\mathrm{SrTiO}_{3}\right)$. Polar nanoregions (PNRs) can naturally exist in perovskitestructure $\mathrm{SrTiO}_{3}$. Due to dimensional engineering for polarization stability, the electrically induced alignment of PNRs leads to a stable net ferroelectric polarization. Therefore, the authors proposed a brand new way for developing roomtemperature ferroelectricity in nanoscale. These approaches can be applied to emerging nanoelectronic devices.

Ferroelectric materials exhibit nonlinear characteristics. Understanding the nonlinear properties in a ferroelectric film for various applications is especially topical [7,8]. Polarization switching is a key issue for nonlinear properties and thereby hinders the possible applications of ferroelectric-material field effect transistors. At the Lawrence Berkeley National Laboratory, University of California, Berkeley, researchers first observed the negative capacitance of ferroelectric $\mathrm{Pb}\left(\mathrm{Zr}_{0.2} \mathrm{Ti}_{0.8}\right) \mathrm{O}_{3}$ (PZT) film (thickness of $60 \mathrm{~nm}$ ) [4]. Using a ferroelectric capacitor and a resistor, the researchers applied voltage pulses across the R-C series circuit and observed the time dynamics of ferroelectric polarization. Negative capacitance corresponds to low-power applications because a low gate voltage can be applied in negative-capacitance transistors. A negative-capacitance field effect transistor (NC-FET) with a ferroelectric material offers ultrafast performance and low power consumption. Power dissipation and overheating are bottlenecks in transistor miniaturization. A low gate voltage can be effective to solve these difficulties. These approaches may be used for developing a next-generation ultrafast transistor with low-power consumption. Although, the switching time measured experimentally is larger than the theoretical results in the current ferroelectric capacitor, NC-FET may present a potential direction for developing $\mathrm{THz}$ logic devices. However, further investigations are necessary to reveal the switching dynamics of improper ferroelectrics.

Ferroelectric materials can also be used for energy generation and conversion [5,9,10], e.g., cryogenic cooling devices based on ferroelectrics. Solid alternatives to hazardous gases in conventional refrigeration applications are being considered. Caloric effect involves a lot of materials, such as ferroelectrics, magnetic and ferroelastics. However, the realized refrigerant efficiency is insufficient for commercial applications because phase transitions are of the first order in most solid-state refrigeration materials. According to a recent theoretical report [5], ultrathin ferroelectric $\mathrm{SrRuO}_{3} / \mathrm{BaTiO}_{3}$ (BTO)/SrRuO 3 (SRO) capacitors show a significantly high caloric effect at room temperature. The phase transition is second order with temperature change over $10 \mathrm{~K}$ at near room temperature. The results suggest high-efficiency nanorefrigeration and potential applications in nanoelectronics.

It must be mentioned that coupling properties play a key role in multifunctions of room-temperature ferroelectric materials. The recent high-quality research papers cited above show that coupling properties not only lead to new physical phenomena, but also facilitate the development of new types of functional devices. These approaches are important for potential applications in ultrafast and low-power nanodevices. Thus, the field of room-temperature ferroelectric materials and their applications is now a hot topic in materials science and electronics engineering.

1 Mandal P, Pitcher M J, Alaria J, et al. Designing switchable polarization and magnetization at room temperature in an oxide. Nature, 2015, 525: 363-366

2 Khan A I, Chatterjee K, Wang B, et al. Negative capacitance in a ferroelectric capacitor. Nat Mater, 2015, 14: 182-186

3 Lee D, Lu H, Gu Y, et al. Emergence of room-temperature ferroelectricity at reduced dimensions. Science, 2015, 349: 1314-1317

4 Becher C, Maurel L, Aschauer U, et al. Strain-induced coupling of electrical polarization and structural defects in $\mathrm{SrMnO}_{3}$ films. Nature Nanotechno, 2015, 10: 661-665

5 Liu Y, Infante I C, Lou X, et al. Giant room-temperature elastocaloric effect in ferroelectric ultrathin films. Adv Mater, 2014, 26: 6132-6137

6 Nan C W, Bichurin M I, Dong S, et al. Multiferroic magnetoelectric composites: historical perspective, status, and future directions. J Appl Phys, 2008, 103: 031101

7 Settera N, Damjanovic D, Eng L, et al. Ferroelectric thin films: Review of materials, properties, and applications. J Appl Phys, 2006, 100: 051606

8 Zhou G Z, Wang Y X, Liu C, et al. On ferroelectric domain polarization switching mechanism subject to an external electric field by simulations with the phase-field method. Sci China Tech Sci, 2013, 56: 1129-1138

$9 \mathrm{Hu} \mathrm{Y,} \mathrm{Wang} \mathrm{Z} \mathrm{L.} \mathrm{Recent} \mathrm{progress} \mathrm{in} \mathrm{piezoelectric} \mathrm{nanogenerators} \mathrm{as}$ a sustainable power source in self-powered systems and active sensors. Nano Energy, 2014, 14: 3-14

10 Han M D, Liu W, Zhang X S, et al. Investigation and characterization of an arc-shaped piezoelectric generator. Sci China Tech Sci, 2013, 56: 2636-2641 